\title{
Descripción de dos nuevas especies de monogéneos microcotiloideos, parásitos de peces marinos de la costa noreste de Venezuela*
}

\author{
JOSÉ LUIS FUENTES ZAMBRANO \\ Departamento de Acuacultura, Escuela de Ciencias Aplicadas del Mar, Núcleo de Nueva Esparta, Universidad de Oriente \\ (apartado postal: 147), Venezuela.
}

\begin{abstract}
RESUMEN: Se describen dos nuevas especies de monogéneos, encontrados en branquias de peces marinos del noreste de Venezuela: Kannaphalus mochimae hallada en Acanthurus coeruleus (Acanthuridae) y Allopyragraphorus marinae en Strongylura marina (Belonidae). K. mochimae difiere respecto a K. virilis Unnithan, 1957 en la forma y disposición de las genitalias; número de grapas y testículos (K. mochimae posee 69 a 76 y de 15 a 20 respectivamente, mientras que $K$. virilis posee 33 a 46 y más de 100 respectivamente); en relación a K. lateriporis Mamaev, 1988 éste posee la vagina de estructura y forma diferente, las grapas son simétricas y el órgano copulatorio posee un corto estilete. En cuanto a K. univaginalis Ramalimgam, 1960 en poseer esta un solo poro vaginal, dos en K. mochimae. A. marinae discrepa de las tres especies hasta ahora señaladas en el género en: talla corporal, forma del opistohaptor, número de grapas, disposición y número de testículos y en la forma y disposición del ovario. Se presenta la clave de los géneros Kannaphalus y Allopyragraphorus.
\end{abstract}

Palabras claves: Kannaphalus, Allopyragraphorus, Microcotyloidea, Monogenea, peces marinos, Venezuela, Sur América.

SUMMARY: DESCRIPTION OF TWO NEW SPECIES OF MICROCOTILOIDS, MONOGENEAN PARASITES IN MARINE FISH FROM THE NORTH EASTERN COST OF VENEZUELA. - Two new species monogenean, found in gills of marine fish from the north easter cost of Venezuela are described: Kannaphalus mochimae found in Acanturus coeruleus (Acanthuridae) and Allopyragraphorus marinae found in Strongylura marina (Belonidae). K. mochimae differs from K. virilis Unnithan, 1957 in shaped and location of the genitals and number of clamps and testis (K. mochimae posses 69 to 76 and 15 - 20 respectively, while $K$. virilis has 33 at 46 and more than 100, respectively). On the order hand, K. lateriporis Mamaev, 1988, another related species, has different structure and form of the vagina, asymmetrical clamps and copulatory organ displaying a short styled. K. univaginalis Ramalimgam, 1960, presents only one vaginal pore, while K. mochimae presents two pore. A. marinae differs from the other three described species of genus in: body size, form of the opisthaptor, number of clamps, number and arrangement of testis, and shaped and arrangement of the ovary. Key to the species of the genera Kannaphalus and Allopyragraphorus are provided.

Key words: Kannaphalus, Allopyragraphorus, Microcotyloidea, Monogenea, marine fishes, Venezuela, South America.

\section{INTRODUCCIÓN}

Los monogéneos resultan de particular importancia patológica cuando afectan a poblaciones de peces

\footnotetext{
*Received 6 de noviembre de 1996. Accepted 24 de abril de 1997.
}

sometidas a cautiverio (Hoffman y Schubert, 1984), considerando a aquellos monogéneos que frecuentan las branquias de peces como los de mayor riesgo, dada la fragilidad y el contacto directo de este órgano con el medio acuático, de ahí que la acción sobre las branquias afecta en primera instancia la respiración de los peces y en última su integridad. 
Para Venezuela a través de la literatura mundial, han sido señaladas 14 especies de monogéneos infestantes de peces marinos. Nasir y Fuentes (1983) describen cuatro nuevas especies, i. e. Allogastrocotyle bivaginalis y Pseudaxine cariacoensis en branquias de Trachurus lathami y Kuhnia scombercolias en Scomber colias; además redescriben a Bicotylophora trachinoti (MacCallum, 1921), 1936 en Trachinotus carolinus; Kuhnia scombri (Kuhn, 1829) Sproston, 1845 en Scomber colias y Gastrocotyle trachuri v. Beneden and Hesse, 1863 en branquias de Trachurus lathami. Bashirullah y Rado (1986) realizan estudios sobre la ocurrencia de tres especies de Choricolyle encontradas en Orthopristes ruber. Fuentes y Nasir (1990) realizan la redescripción y distribución espacial de Ligophorus mugilinus (Hargis, 1955) Euzet y Su-riano, 1977 parásito branquial de Mugil curema capturadas en la Laguna de La Restinga. Bashirullah y Rodríguez (1992) determinan la distribución espacial en las branquias de Caranx hippos de Protomicrocotyle mirabilis (MacCallum, 1919) Jhonston et Tigs, 1922; Cemocotyle noveboracensis Price 1962; Allopyragraphorus hippos (Hargis, 1956) Yamaguti, 1963 y Cemocotyllela elongata (Meserve, 1938) Price, 1962. Fuentes y Ochoa (1993) proponen un nuevo género y una nueva especie Neomurraytrematoides proops parásito del bagre Arius proops. Varias de estas especies de hospederos son cultivados o representan potencialidad para ello, de ahí la importancia de conocer factores de índole biológica que puedan interferir en el desarrollo de su cultivo.

En el presente trabajo se describen dos nuevas especies de monogéneos microcotiloideos encontrados en peces marinos en dos sistemas litorales del oriente de Venezuela.

\section{MATERIAL Y MÉTODOS}

Los peces se capturaron con una red de $25 \mathrm{~m}$ de longitud, en localidades de la Bahía de Mochima $\left(10^{\circ} 21^{\prime}\right.$ y $10^{\circ} 24^{\prime}$ de latitud norte y $64^{\circ} 19^{\prime} 33^{\prime \prime}$ y $64^{\circ} 22$ ' 30" de longitud oeste) y la Laguna La Restinga $\left(10^{\circ} 57^{\prime}\right.$ y $11^{\circ} 03^{\prime}$ de latitud norte y $64^{\circ}$ $01^{\prime}$ y $64^{\circ} 12^{\prime}$ de longitud oeste) ambas ubicadas al noreste de Venezuela. Las capturas se realizaron con una frecuencia bimensual, durante enero a mayo de 1996. Los peces fueron trasladados vivos al laboratorio, donde una vez sacrificados, se les disecó para extraerles las branquias, previa observación superficial de la piel y raspado de la misma. En el caso de las branquias, fueron separados los distintos arcos y luego colocados en cápsulas de Petri conteniendo solución salina al 0,75 $\%$ o agua de mar filtrada, mientras que los raspados de piel fueron colocados en portaobjetos. Los parásitos encontrados, fueron extraídos con pipetas, muertos por calor y fijados por los métodos aplastamiento lámina-lámina o lámina-laminilla e inclusión en Bouin o formalina al 5\%, donde permanecieron por espacio de 6 a 12 horas. La preservación se realizó con etanol al $70 \%$ por espacio de 30 minutos. Seguidamente los organismos fueron teñidos utilizando los métodos de Aceto Carmín de Semichon o Hematoxilina de Harris. Luego se deshidrataron y aclararon en aceite de clavo. Por último fueron montados permanentemente en Bálsamo de Canadá. También se realizaron observaciones de los individuos mediante preparados acuo-sos, tiñéndolos con rojo neutro o azul de metileno, lo cual algunas veces permitió discernir estructuras difíciles de ver en preparaciones permanentes.

Las descripciones y dibujos se realizaron con la ayuda de microscopio óptico y la cámara clara, agregando ciertos detalles de los dibujos a mano alzada. Las medidas se efectuaron mediante micrómetro ocular y se expresan en milímetros, encerrando entre paréntesis el promedio de las mismas y ordenadas de tal forma que primero se indica la realizada en el eje longitudinal y a continuación la tomada del eje transversal. La longitud del cuerpo incluye al opistohaptor y la medida del ancho del cuerpo se realizó a nivel de la zona ovárica.

\section{RESULTADOS Y DISCUSIONES}

Superfamilia: MicrocotYLOIDEA Unnithan, 1957 Familia: HETERAXINIDAE Price, 1962

Subfamilia: Heteraxininae Unnithan, 1957 Género: Kannaphalus Unnithan, 1957

Kannaphalus mochimae sp. nov. (Fig. 1)

Hospedador: Acanthurus coeruleus (Acanthuridae)

Nombre local: " navajón "

Localización: filamentos branquiales

Localidad geográfica: Puerto de Guaigua, Bahía de Mochima, Venezuela

Rango de la lt de los ejemplares examinados: 152 - $227 \mathrm{~mm}$ 


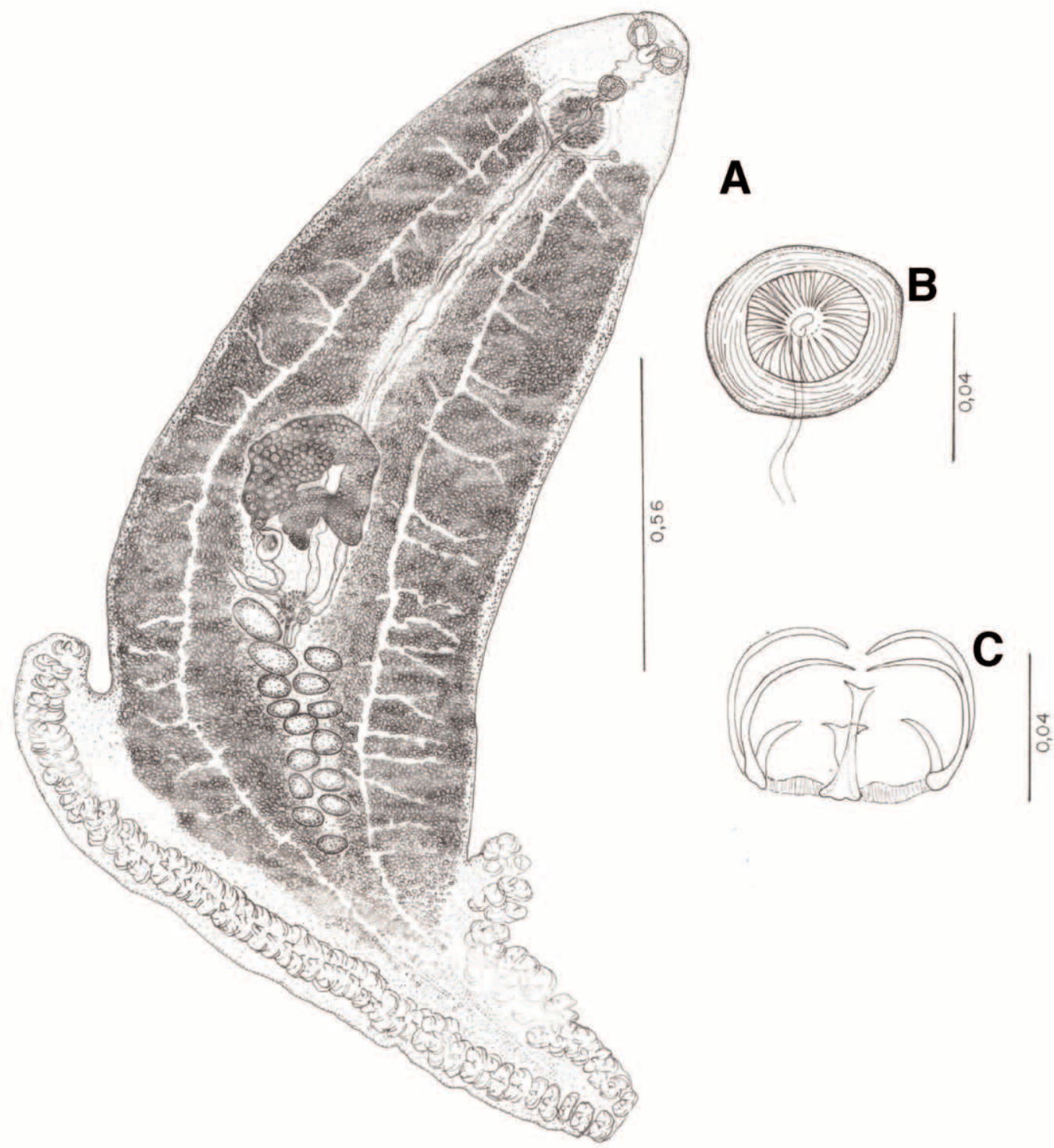

FIG. 1. - Kannaphalus mochimae sp. nov. (valores de las escalas en mm). A) holotipo, verme completo, vista ventral; B) bulbo eyaculatorio de paratipo; C) grapa del holotipo

FIG. 1. - Kannaphalus mochimae sp. nov. (scales in mm). A) holotype, entire worm, ventral view: B) eyaculatori bulb of paratype; C) clamp of the holotype.

$\mathrm{N}^{\mathrm{o}}$ de hospedadores examinados: 15

Prevalencia: $66,67 \%$

Intensidad: 2 - 4

Intensidad media: 3

\section{Descripción de la especie}

Las descripciones y medidas (en mm) están basadas en seis ejemplares.

Cuerpo de forma triangular, atenuado en el extremo anterior. Medidas 1,511 a 1,990 (1,714) de lon- gitud por 0,556 a $0,733(0,659)$ de anchura máxima. Prohaptor constituido por dos ventosas semicirculares, subterminales de 0,040 a $0,051(0,046)$ por 0,032 a $0,046(0,039)$. Opistohaptor asimétrico respecto al eje longitudinal del cuerpo, constituido por un cotilóforo de dos hileras de grapas, de las cuales la derecha de 1,156 a $1,378(1,237)$ de longitud y con 47 a 51 grapas, está significativamente más desarrollada que la izquierda que tiene una longitud de 0,489 a $0,578(0,536)$ y con 22 a 25 grapas. Grapa de 0,058 a $0,070(0,062)$ por 0,040 a $0,058(0,053)$; 
con esqueleto grapal simétrico de tipo microcotile, constituido por tres pares de escleritos laterales y una pieza media con dos brazos, ambos de extremos libres bifurcados. Lóbulo y ganchos opistohaptorales ausentes.

Boca subterminal. Faringe oval o piriforme de 0,040 a $0,041(0,041)$ por 0,026 a $0,032(0,030)$. Esófago corto y diverticulado. Intestino bifurcado, diverticulado, ciegos intestinales no se unen posteriormente, el ciego izquierdo de mayor longitud que el derecho pero ambos penetran al opistohaptor.

Testículos ovalados de 0,040 a $0,041(0,041)$ por 0,052 a $0,062(0,052)$; en número de 15 a 20 , intercrurales, preováricos, dispuestos transversalmente al eje del cuerpo. Vaso deferente mediano, con marcadas sinuosidades en el tercio anterior antes de terminar en un órgano copulador no diferenciado. Ducto eyaculotorio provisto en la base de un bulbo cirral muscular de 0,034 de diámetro desde donde se originan 28 espinas genitales de extremo libre curvo, dirigido a la luz de la abertura del ducto. Atrio genital se localiza ventralmente, la abertura está rodeada de un esfínter de pared gruesa. Poro genital de 0,062 a 0,064 de diámetro. Glándulas prostáticas agrupadas en dos áreas semicirculares, inmediatamente posteriores al atrio genital, el cual se encuentra a una distancia de 0,104 a $0,106(0,105)$ del extremo anterior del cuerpo.

Ovario preecuatorial, pretesticular, doblado, con los extremos dirigidos hacia la parte posterior del cuerpo. Oviducto descendente, antes de proyectarse al ootipo sufre una ligera contorsión. Receptáculo seminal semiesférico de 0,046 por 0,052 , con nacimiento al inicio del oviducto, para luego solaparlo ventralmente. Dos grupos de células glandulares de Mehlis se localizan en el extremo anterior del ootipo el cual tiene forma oval y mide 0,049 a 0,058 por 0,032 a 0,036 . Útero mediano, tiene origen en el extremo anterior del ootipo. Dos poros vaginales de 0,016 de diámetro, submedianos dorsales, posterolaterales a la bifurcación intestinal; los conductos vaginales discurren hasta la parte media del cuerpo, para constituir un conducto único en el final posterior de las áreas glandulares prostáticas y desciende hasta coincidir con el reservorio vitelogénico. Canal genitointestinal desemboca en el ciego intestinal derecho después de proyectarse desde el extremo terminal del ootipo. Conducto vitelogénico en forma de "Y", vitelaria folicular y coextensiva con el intestino, excepto en el extremo anterior y posterior del mismo. No se observaron huevos.

\section{Discusión}

Price (1962) creó la Familia Heteraxinidae, para agrupar en ella aquellas especies que anteriormente se les ubicaba en Microcotylidae Taschenberg, 1879, considerando como característica diagnóstica diferencial el hecho de que poseen un opistohaptor asimétrico, triangular, constituido por dos filas de grapas de longitudes desiguales.

La presencia de grapas en los dos lados o caras del opistohaptor, la simetría de la grapa y la estructura típica de microcotiloide, así como la ausencia de fosa dorsal permite ubicar al organismo estudiado en la Subfamilia Heteraxininae Unnithan, 1957 y en el Género Kannaphalus Unnithan, 1957.

La especie estudiada y las hasta ahora señaladas en Kannaphalus, poseen una particularidad relacionada con la presencia de un bulbo muscular (bulbo eyaculatorio) o, como lo denomina Mamaev (1988), un bulbo atrial, en la base del ducto eyaculatorio ('penis gum' según Unnithan, 1957). Este órgano, en la especie de Kannaphalus estudiada, esta provisto de 28 espinas genitales concéntricas alrededor de la abertura genital masculina.

Hasta el momento se han señalado tres especies a nivel mundial como pertenecientes Kannaphalus, son: K. virilis Unnithan, 1957, especie tipo, encontrada en Caranx atropus; K. lateriporis Mamaev, 1988 en C. sexfasciatus, ambas teniendo como localidad geográfica el sur de la India, y Rama-limgam (1960) refiere a $K$. univaginalis en $C$. sexfasciatus en el mismo país, pero su descripción no está lo suficientemente documentada, hasta el punto de que Yamaguti (1963) no la incluye en su clave. Sin embargo, la posibilidad de igual identidad entre $K$. univaginalis con la especie en estudio queda descartada, al poseer, la primera una única abertura vaginal.

La nueva especie propuesta difiere de $K$. virilis en: a) talla corporal, siendo $K$. virilis de mayor tamaño; b) número de grapas en cada cotilóforo: en la especie sometida a comparación se encuentran de 21 a 31 en el lado derecho y de 12 a 15 en el izquierdo, mientras que en la especie estudiada son significativamente más numerosas, 47 a 51 y 22 a 25 , respectivamente; c) número de testículos: $K$. virilis posee más de 100, mientras que la nueva especie propuesta posee de 15 a 20 ; d) disposición y forma de la genitalia femenina y e) otras discrepancias relacionadas con la disposición del sistema reproductor masculino. 
TABLA 1. - Algunas comparaciones entre Kannaphalus mochimae sp. nov. , K. virilis y K. lateriporis.

TABLE 1. - Some comparisons between Kannaphalus mochimae sp. nov., K. virilis and K. lateriporis.

\begin{tabular}{|c|c|c|c|}
\hline & K. mochimae* & K. virilis + & K. lateriporis ${ }^{\circ}$ \\
\hline Talla & $\begin{array}{c}1,511 \text { a } 1,990 \\
\text { por } \\
0,556 \text { a } 0,733\end{array}$ & $\begin{array}{c}3,500 \text { a } 3,600 \\
\text { por } \\
0,825 \text { a } 0,845\end{array}$ & - \\
\hline $\mathrm{N}^{\mathrm{o}}$ de Grapas & $\begin{array}{l}\text { Cotilóforo der. } 47 \text { a } 51 \\
\text { Cotilóforo izq. } 12 \text { a } 5\end{array}$ & $\begin{array}{l}\text { Cotilóforo der. } 21 \text { a } 31 \\
\text { Cotilóforo der. } 20 \text { a } 32\end{array}$ & $\begin{array}{l}\text { Cotilóforo izq. } 22 \text { a } 25 \\
\text { Cotilóforo izq. } 13 \text { a } 16\end{array}$ \\
\hline Grapas & Simétrica & Simétrica & Asimétrica \\
\hline Poros vaginales & (2) Submedianos dorsales & (2) Submedianos dorsales & (2) Dorsolaterales \\
\hline Testículos & $12-20$ & Más de 100 & - \\
\hline Órgano copulatorio & Tubular, ausencia de estilete & Tubular, ausencia de estilete & Tubular, estilete corto presente \\
\hline Bulbo atrial & Más grande que la faringe & Más grande que la faringe & Más pequeño que la faringe \\
\hline Hospedador & Acanthurus coeroleus & Caranx atropus & Caranx sexfaciatus \\
\hline Localización Geográfica & Bahía de Mochima, Venezuela & Sur de la India & Sur de la India \\
\hline
\end{tabular}

* Datos compilados de ejemplares de la presente investigación.

+ Datos compilados de Unnithan, 1957

o Datos compilados de Mamaev, 1988

Las siguientes características presentes en $K$. lateriporis permiten diferenciarla de la especie estudiada: estructura y posición de la vagina (abertura dorsolateral), la asimetría de la grapa, la presencia de un estilete sobre el órgano copulatorio y el pequeño tamaño del bulbo cirral.

Para otras comparaciones entre Kannaphalus mochimae con $K$. virilis y $K$. lateriporis ver Tabla 1.

Etimología: se refiere a la localidad geográfica donde fueron recogidos los hospedadores, la Bahía de Mochima.

Clave de las especies del género Kannaphalus

1. - Un solo poro vaginal K. univaginalis Rama-limgam, 1960

- Dos poros vaginales 2

2. - Estilete cirral presente K. lateriporis Mamaev, 1988

- Estilete cirral ausente

3. - Más de 100 testículos K. virilis Unnithan, 1957

- De 15 a 20 testículos ........ mochimae sp. nov.
Familia AlLOPYRAGRAPHORIDAE Yamaguti, 1963 Género Allopyragraphorus Yamaguti, 1963

Allopyragraphorus marinae sp. nov. (Fig. 2)

Hospedador: Strongylura marina (Belonidae)

Nombre local: 'marao'

Localización: filamentos branquiales.

Localidad: La Tortuga y Giele, Laguna de La Restinga, Venezuela

Rango de la lt de los ejemplares examinados: 227 $-413 \mathrm{~mm}$

$\mathrm{N}^{\mathrm{o}}$ de hospedadores examinados: 20

Prevalencia: $60 \%$

Intensidad: 1 - 4

Intensidad media: 1,50

\section{Descripción de la especie}

Las descripciones y medidas (en mm) están basadas en ocho ejemplares.

Cuerpo alargado con el extremo anterior acusado y el posterior ancho de 2,020 a $2,244(2,16)$ de 


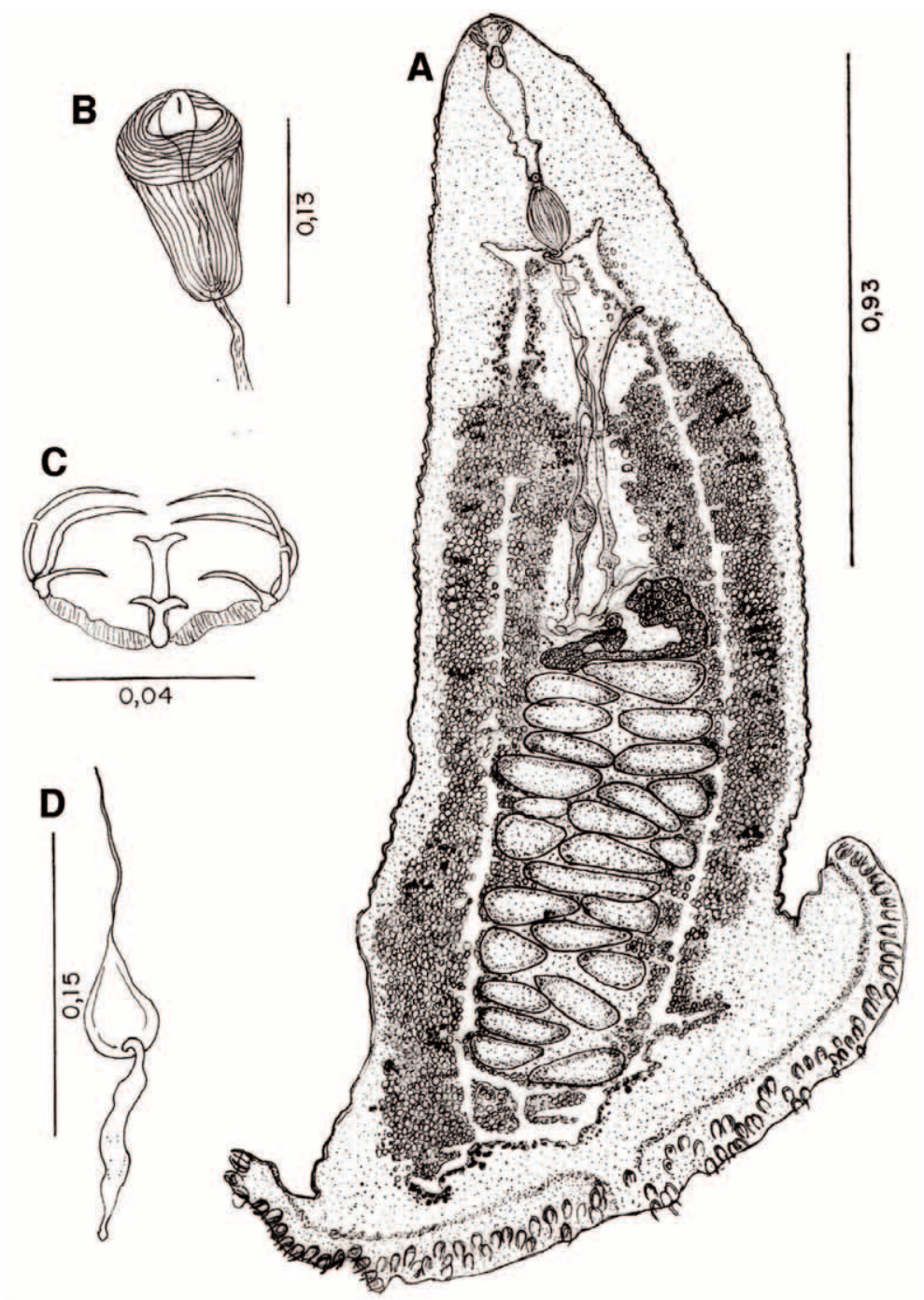

FIG. 2. - Allopyragraphorus marinae sp. nov. (valores de las escalas en mm). A) holotipo, verme completo, vista dorsal; B) genitalia masculina terminal de paratipo; C) grapa de paratipo; D) huevo del holotipo.

FIG. 2. - Allopyragraphorus marinae sp. nov. (scales in mm). A) holotype, entire worm, dorsal view; B) terminal genitalia male of paratype; C) clamp of paratype; D) egg of the holotype.

longitud por 0,533 a $0,867(0,680)$ de ancho. Prohaptor constituido por un par de ventosas de 0,030 a $0,058(0,038)$ por 0,018 a $0,022(0,020)$, que abre en el interior de la cavidad bucal, dispuesta oblicuamente y anterolaterales a la faringe. Opistohaptor en forma de aleta caudal de pez, ligeramente simétrico, con una longitud transversal de 0,909 a 1,390 (1,150); constituido por dos filas de grapas separadas entre sí por una constricción inconspicua. El número de grapas del cotilóforo derecho varia entre 39 y 42 , el izquierdo entre 31 y 38 . Las grapas laterales de menor tamaño que las medianas de 0,024 a $0,040(0,027)$ por 0,020 a $0,040(0,033)$ y 0,022 a $0,048(0,033)$ por 0,024 a $0,076(0,052)$, respectivamente. Todas las grapas son de tipo microcotile, con tres pares de escleritos laterales arqueados y un esclerito mediano de dos ramas de extremos terminales bifurcados. Lóbulo y ganchos del opistohaptor ausentes.

Boca subterminal. Faringe piriforme de 0,032 a $0,044(0,035)$ por 0,018 a $0,020(0,020)$. Esófago ancho y ligeramente diverticulado con una longitud de 0,138 a $0,342(0,265)$. Intestino diverticulado lateralmente, bifurcado, distancia prebifurcal de 0,243 a $0,441(0,369)$, unido posteriormente mediante doble anastomosis y penetrando parcialmente el opistohaptor.

Testículos foliculares de 0,054 a $0,160(0,100)$ por 0,032 a $0,064(0,047)$ en número de 16 a 25 , post-ováricos, dispuestos en dos filas intercecales desde el 
extremo inmediato posterior del ovario hasta la unión de los ciegos intestinales. Vaso deferente mediano, de tortuoso recorrido antes de penetrar a la bolsa del órgano copulador, la cual es oval y mide de 0,080 a $0,120(0,105)$ por 0,054 a $0,094(0,074)$; cons-tituida por fibras musculares longitudinales. Pene muscular de 0,042 a $0,046(0,044)$ de largo, desarmado, evaginable, el inicio es tubular para luego terminar en una ampliación en forma de lengüeta.

Ovario preecuatorial, tubular, el tercio medio es transversal al eje del cuerpo, mientras que los extremos se doblan por separado, el tercio inicial lo hace dos veces. Ootipo submediano izquierdo. Oviducto corto. Receptáculo seminal semiesférico de 0,096 a 0,062, localizado inmediatamente anterior o solapado dorsalmente por la rama inicial del ovario.útero mediano, con recorrido ventral a la bolsa del pene para desembocar en un poro genital común, ventral y prebifurcal. Canal genitointestinal comunica con el ciego derecho después de proyectarse oblicuamente desde el conducto vitelogénico. Poro vaginal dorsal, submediano derecho, posterolateral a la bifurcación intestinal. Reservorio vitelogénico en forma de ' $\mathrm{Y}$ '. Vitelaria folicular distribuida desde el inicio del tercio medio del cuerpo hasta disminuir paulatinamente al final de los ciegos intestinales. Huevos con dos filamentos, uno en cada extremo.

\section{Discusión}

Yamaguti (1963) en su compendio creó el género Allopyragraphorus, diferenciándolo de Pyragraphorus Sproston, 1946, el más afín, en función de las siguientes características: la uniformidad del tipo de grapa del opistohaptor, la unión posterior de los ciegos intestinales y la ausencia de espinas en el cirro; proponiendo así mismo, para el género creado, tres nuevas combinaciones, especie tipo, Allopyragraphorus hippos (Hargis, 1956) (sin. Pyragraphorus hippos); A. caballeroi (Zerecero 1960) (Pyragraphorus caballeroi) y A. incomparabilis (MacCallum, 1917) (sin. Microcotyle incomparabilis). Todas las especies parasitan branquias de Caranx hippos, aun cuando $A$. incomparabilis fue también hallado en las branquias de C. ruber.

La nueva especie propuesta difiere principalmente de las hasta ahora integrantes de Allopyragraphorus en: talla corporal; forma del opistohaptor, A. hippos y A. incomparabilis poseen el lado izquierdo del órgano de anclaje visiblemente más desarrollado que el derecho; número de grapas; disposición y número de testículos, en la especie estudiada los testículos son ovalados, dispuestos mayoritariamente en el eje longitudinal perpendicular al eje del cuerpo, en las

TABLA 2. - Algunas comparaciones entre Allopyragraphorus marinae sp. nov. y las especies del género hasta ahora descritas.

TABLE 2. - Some comparisons between Allopyragraphorus marinae sp. nov. and presently described species of the genus.

\begin{tabular}{|c|c|c|c|c|}
\hline & A. marinae* & A. hippos + & A. incomparabilis \# & A. caballeroi $^{\circ}$ \\
\hline Talla corporal & $\begin{array}{c}2,020-2,240 \\
\text { por } \\
0,533-0,867\end{array}$ & $\begin{array}{c}2,90-3,30 \\
\text { por } \\
0,70-1,00\end{array}$ & 5,00 por 1,80 & 3,38 por 4,86 \\
\hline $\mathrm{N}^{\mathrm{o}}$ total de grapas & 70 a 79 & 55 a 60 & 86 a 98 pares & 67 a 104 \\
\hline Opistohaptor & $\begin{array}{l}\text { Ligeramente asimétrico, } \\
\text { constricción presente. }\end{array}$ & $\begin{array}{l}\text { Asimétrico, } \\
\text { constricción presente. }\end{array}$ & $\begin{array}{l}\text { Asimétrico, } \\
\text { constricción presente. }\end{array}$ & $\begin{array}{l}\text { Ligeramente asimétrico, } \\
\text { constricción presente. }\end{array}$ \\
\hline Testículos & 16 a 23 & Aprox: 50 & Aprox.: 75 & Más de 100 \\
\hline Ovario & $\begin{array}{l}\text { Doblado, con los extremos } \\
\text { dirigidos hacia adelante. }\end{array}$ & $\begin{array}{l}\text { Doblado con los extremos } \\
\text { dirigidos hacia atrás. }\end{array}$ & $\begin{array}{l}\text { Doblado con los extremos } \\
\text { dirigidos hacia atrás. }\end{array}$ & $\begin{array}{l}\text { Doblado con los extremos } \\
\text { dirigidos hacia atrás. }\end{array}$ \\
\hline Poro vaginal & Submediano derecho & Mediano & Mediano & Mediano \\
\hline Hospedador & Strongylura marina & Caranx hippos & $\begin{array}{l}\text { Caranx hippos y } \\
\text { Caranx ruber }\end{array}$ & Caranx hippos \\
\hline Loc. geográfica & $\begin{array}{l}\text { Isla de Margarita, } \\
\text { Venezuela }\end{array}$ & Florida, USA. & Manzanillo, México. & Florida, USA. \\
\hline
\end{tabular}

* Datos compilados de los ejemplares de la presente investigación

+ Datos compilados de Hargis (1956)

\# Datos compilados de MacCallum (1917)

o Datos compilados de Zerecero (1960) 
tres especies comparadas los testículos son semiesféricos; disposición de la genitalia; forma y disposición del ovario, en la nueva especie propuesta los extremos del ovario están orientados hacia la parte anterior del cuerpo mientras que en las restantes lo hacen hacia el extremo posterior.

En la Tabla 2 se señalan otras comparaciones entre A. marinae y otros integrantes del género hasta ahora descritos.

Etimología: El nombre específico de la nueva especie hace referencia al nombre científico del hospedador en el que fue hallada.

\section{Clave de las especies del género Allopyragraphorus}

1.- Extremos del ovario orientados hacia la parte anterior del cuerpo ...... A. marinae sp. nov.

- Extremos del ovario orientados hacia la parte posterior del cuerpo................. 2

2. - Más de 100 testículos A. caballeroi (Zerecero, 1960)

- Menos de 100 testículos ............... 3

3.- Número de grapas de 86 a 98 pares A. incomparabilis (MacCallum., 1917)

- Número de grapas de 55 a 60 A. hippos (Hargis, 1956)

\section{BIBLIOGRAFÍA}

Bashirullah, A. and Rado, N. - 1987. Co-ocurrence of three species of Choricotyle (Monogenea) in the grunt, Orthopristis ruber and host specificits. J. Fish. Biol., 30: 419-422.

Bashirullah, A. and Rodríguez, J. - 1992. Spatial distribution and interrelationship of four monogenoidea of jack mackerel, Caranx hippos (Carangidae) in the north-easth of Venezuela. Biol. Mar. Acata Cient. Venezolana, 43: 125-128.

Fuentes, J. L. and Nasir, P. - 1990. Descripción y ecología de Ligophorus mugilinus (Hargis, 1955) Euzet y Suriano, 1977 (Monogenea, Ancyrocephalinae) en Mugil curema (Val. 1936) de la Isla de Margarita, Venezuela. Scient. Mar., 54: 187-193.

Fuentes, J. L. y Ochoa, G. - 1993. Neomurraytrematoides proops gen. nov., sp. nov. (Monogenea): parásito branquial de Arius proops (Pices, Ariidae) de la Isla de Margarita, Venezuela. Ciencias, 1: 9-12.

Hargis, W. - 1956. Monogenetic trematodes of Gulf of México fishes. Part X. The Family Microcotylidae Taschenberg, 1879. Tr. Am. Micr. Soc. 75: 436-454.

Hoffman, G. and Schubert, G. - 1984. Some parasites of exotic fishes. Distribution, Biology and Management of Exotic Fishes. J. H. Univ. Press. (De.), London: 233-261

MacCallum, G. A. - 1917. Some new forms of parasitic worms. Zoopathologica, 1: 43-75.

Mamaev. Y. - 1988. Kannaphalus lateriporis n. sp. and its position within the system of higher monogeneas. Parasitologiya, 22: 354-358.

Nasir, P. and Fuentes, J. L. - 1983. Algunos tremátodos monogenéticos venezolanos. Riv. Parassit., 44: 355-380.

Price, E. W. - 1962. North American monogenetic trematodes, XI. The Family Heteraxinidae. J. Parasit., 48: 402-418.

Ramalimgam, K. - 1960. The functional development of 'compensating asymmetry' in the higher Monogenea. Wiss. zool. 164: 374-381.

Unnithan, V. - 1957. On the functional morphology of a new fauna of monogenea of fish from Trivandrum and environs. Part I. Axinidae fam. nov. Bull. Centr. Research Inst. Univ. Kerala 5: 27-122.

Yamaguti, S. - 1963. Sistema Helminthum. Monogenean and Aspidocotylea. Vol. IV. Intersc. Publ. Nueva York, $699 \mathrm{pp.}$

Zerecero, D. M. - 1960. Pyragraphorus caballeroi n. sp. (Trematoda de la subclase Monogenea Carus, 1763) en: Peces marinos del Oceano Pacífico del Norte. Libro Homenaje al Dr. Eduardo Caballero, 345-351.

Scient. Ed.: S. Zanuy 